\title{
Monitoramento e Avaliação de Estudantes em Mundos Virtuais
}

Felipe Becker Nunes, PPGIE, UFRGS, nunesfb@gmail.com Fabrício Herpich, PPGIE, UFRGS, fabricio.herpich@ gmail.com

Liane Margarida Rockenbach Tarouco, PPGIE, UFRGS, liane@penta.ufrgs.br José Valdeni de Lima, PPGIE, UFRGS, valdeni@inf.ufrgs.br

Resumo. O processo de monitoramento e avaliação das tarefas educacionais realizadas pelos usuários dentro dos mundos virtuais se trata de um aspecto problemático ao docente, visto que este tipo de suporte genuinamente não é fornecido pelos mundos virtuais. Com base nisto, este artigo apresenta uma proposta de implementação de recursos para realizar o monitoramento dos usuários em mundos virtuais. $\mathrm{O}$ ambiente OpenSim foi utilizado e nele foram agregados recursos como controle de assiduidade, interações dos usuários, avaliação e registro das conversações realizadas pelos alunos. Como resultados, foi constatado a viabilidade desta proposta e o correto funcionamento dos recursos nos testes realizados.

Palavras - Chave: monitoramento, mundos virtuais, educação.

\section{Tracking and Assessment of Student's in Virtual Worlds}

\begin{abstract}
The process of monitoring and evaluation of educational tasks performed by users within the virtual worlds it is a problematic aspect to the teacher, as this type of support is not genuinely provided by virtual worlds. On this basis, this paper presents a proposal for deployment of resources to carry out the monitoring of users in virtual worlds. OpenSim environment was used and there were added features such as attendance control, user interactions, evaluation and recording of conversations held by students. As a result, it was found the viability of this proposal and the correct functioning of the resources in the tests.
\end{abstract}

Keywords: monitoring, virtual worlds, education.

\section{INTRODUÇÃO}

A introdução de novos paradigmas de aprendizagem e metodologias de ensino modificou o cenário tradicional visto nas salas de aula. O uso das Tecnologias da Informação e Comunicação (TICs) na área educacional apresentou novas alternativas de aprendizagem e estendeu o ensino para além do ambiente presencial de sala de aula. Dentre as soluções existentes, estão os ambientes virtuais de aprendizagem e os mundos virtuais, que podem ser utilizados como suporte para a realização de atividades.

Uma definição explicitada por Yilmaz et al. (2015) fornece uma visão mais ampla acerca dos mundos virtuais, também comumente chamados de ambientes imersivos, em que se tratam de espaços 3D onde os usuários interagem constantemente com ambientes agradáveis e realistas, os quais podem até mesmo, refletir as situações de vida diária dos usuários. Quando aplicados no âmbito educacional, estes ambientes podem agregar características importantes às interações dos usuários, como a sensação de imersão, comunicabilidade e interatividade, que podem auxiliar na realização das atividades educacionais propostas. 
Por se tratar de um ambiente que pode ser disponibilizado de forma online, o desenvolvimento de tarefas à distância pode ser executado em qualquer momento e lugar, podendo resultar em um processo de aprendizagem. Rafalski et al. (2014) entendem que os estudantes deixam de ser meros espectadores dos acontecimentos, outrora exclusivos à sala de aula, para serem protagonistas do processo de aprendizagem em ambientes de autoria e cooperação.

Considerando o contexto apresentado, em que a realização de atividades educacionais pode ocorrer na modalidade de ensino a distância, uma problemática se instaura neste processo ao se refletir acerca da forma com que os alunos serão monitorados e avaliados pelo professor. Bouda (2012) explica que os mundos virtuais utilizados na educação estão sendo construídos com enfoques particulares para o aprendizado de conteúdos específicos, porém a avaliação desses mundos ainda ocorre de forma empírica, através da observação da utilização dos MV pelos seus usuários

Genuinamente, este tipo de ambiente não foi desenvolvido exclusivamente para uso educacional, como pode ser constatado em outros tipos de softwares, como o Moodle, que se trata de um ambiente virtual de aprendizagem desenvolvido com foco no âmbito educacional e preparado para realizar o monitoramento e avaliação das atividades educacionais realizados pelos estudantes.

Desta forma, no mundo virtual existem limitações e dificuldades no processo de coletar dados resultantes das interações dos usuários no mundo virtual, como o momento que acessou e saiu do ambiente, quais locais visitou e que atividades desempenhou, assim como a realização de avaliações. Tais exemplos de informações coletadas podem ser considerados essenciais para que o professor possa realizar uma análise consistente de uma intervenção didática ocorrida neste tipo de ambiente.

Diante desta problemática, este artigo apresenta uma proposta de monitoramento das ações e atividades desempenhadas por estudantes dentro do mundo virtual durante a realização de tarefas didáticas. O ambiente OpenSim foi utilizado para realizar a implementação dos recursos propostos, no qual foram realizados testes com alunos para averiguar a viabilidade do ponto de vista tecnológico acerca do processo de coletar informações relevantes sobre as interações dos estudantes no mundo virtual.

\section{TRABALHOS RELACIONADOS}

Com o objetivo de situar o leitor acerca das pesquisas já desenvolvidas envolvendo o escopo deste trabalho, e, esclarecer as diferenças existentes nesta proposta, foram selecionados alguns trabalhos para serem detalhados. No que concerne ao campo da avaliação do aluno em mundos virtuais, o trabalho desenvolvido por Falcade et al. (2014) aborda a criação de uma metodologia de verificação do desempenho do aluno, em que diversas métricas e indicadores foram estabelecidos e testados para verificar sua viabilidade.

Levando em consideração este contexto, também é possível citar os trabalhos desenvolvidos por Antunes, Morgado, Martins, \& Fonseca (2007) e Madeira (2008), em que tratam do controle de assiduidade do aluno no ambiente 3D Second Life. Uma ferramenta foi desenvolvida para realizar automaticamente o registro de presença dos usuários diretamente na base do ambiente Moodle. Desta forma, cada acesso realizado pelo aluno no Second Life estava registrado para posterior consulta do professor.

No que diz respeito a realização de um monitoramento mais aprofundado dos alunos em mundos virtuais, algumas iniciativas têm sido desenvolvidas no decorrer dos últimos anos, como pode ser visto nos trabalhos de Camilleri et al. (2013) e Tibola e Tarouco (2015). Tais pesquisas abordam a questão do sensoriamento, em que as propostas 
apresentadas executam o registro dos alunos no mundo virtual, assim como as salas/laboratórios que visitaram e as datas/horas que foram efetuadas a captura dos dados.

Por fim, a ferramenta Sloodle também está disponível para utilização de forma integrada do mundo virtual com o ambiente Moodle, sendo disponibilizado diversos recursos comuns que são criados em ambos ambientes e utilizados pelo aluno no mundo virtual. Tal processo mantém o registro das interações realizadas no Moodle, como o uso do "tracker", que registra a presença do aluno no mundo virtual, além das conversas oriundas do chat que são armazenadas no Moodle.

Contextualizado os recursos e iniciativas propostas por diferentes autores no decorrer dos últimos anos, torna-se possível esclarecer as diferenças pertinentes presentes nesta proposta. Sua diferença está pautada no número de recursos de sensoriamento disponibilizados para o professor, em que são coletadas informações detalhadas das interações dos alunos no mundo virtual.

Tais informações são exemplificadas como o controle de assiduidade e detalhado das atividades que desempenhar neste ambiente, as notas provenientes dos questionários respondidos e ações realizadas em tempo real, que detectam que uma determinada situação do aluno, como estar sem interagir no mundo virtual e emite um comunicado ao usuário tentando motivá-lo. Além disso, trata-se de uma proposta independente do ambiente do Moodle, podendo ser utilizada de forma individual, diferentemente do Sloodle.

\section{MUNDOS VIRTUAIS}

Os mundos virtuais são ambientes que simulam diversos objetos do mundo real de forma gráfica tridimensional, os quais buscam transcender um alto grau de realidade para que seus usuários se sintam imersos em seus avatares (representação virtual do usuário) e possam se comunicar e interagir com demais participantes presentes neste tipo de ambiente. Conforme Bulu (2012), os indivíduos podem controlar seus avatares se movimentando, correndo, andando ou voando, além de se comunicar com demais usuários usando gestos, voz e/ou de forma textual por meio do chat.

Dentre os ambientes disponíveis atualmente, pode-se citar o Second Life, Open Wonderland e Open Simulator (OpenSim). Para o escopo deste trabalho, foi selecionado o OpenSim por ser gratuito, open source, com vasta documentação no meio acadêmico e de conhecimento dos autores deste trabalho. O OpenSim destaca-se por ser utilizado na criação e no desenvolvimento de ambientes imersivos em 3D, similar ao Second Life, encontrando-se em constante desenvolvimento e implementação, oferecendo um potencial de uso para a criação de ambientes em 3D (REIS et al., 2013).

Para a visualização dos mundos virtuais, como o OpenSim, se torna necessário realizar a instalação de uma aplicação denominada "viewer", que se trata de um software que deve ser configurado com os parâmetros de conexão ao mundo virtual que se deseja acessar. Conforme Nunes et al. (2013), a escolha de um viewer que não atenda determinadas necessidades pode acarretar em diversos problemas: no caso de um viewer que exige mais processamento, o usuário deverá optar por um hardware mais robusto ou trabalhar com desempenho limitado, podendo causar a insatisfação do mesmo; ou ainda no caso de uma visualização incorreta dos objetos importados, que irá consequentemente limitar as ações deste no mundo, impedindo a realização de determinadas atividades

Após realizado este procedimento, o acesso é realizado por meio de um usuário e senha para interagir com o ambiente 3D. Existem diversas soluções atualmente disponíveis, como Singularity, Imprudence e Firestorm. 
Com relação à disposição espacial dentro do ambiente, Carmo (2013) explica que o termo região no OpenSim, a qual podem ser criadas no formato de pequenos terrenos (256m x $256 \mathrm{~m})$, que podem estar tanto interligados quanto separados geograficamente no Mundo Virtual. Desta forma, a distribuição desses terrenos obedece uma matriz bidimensional ( $\mathrm{X}$ e $\mathrm{Y}$ ) para que os mesmos possam ser posicionados sem que ocorram conflitos de posicionamento.

Conforme descrito na introdução deste trabalho e corroborado pela afirmação de Amaral et al. (2012), os mundos virtuais vêm sendo explorados para os mais diversos fins, quebrando a barreira do campo do entretenimento para se consolidar na área educacional. A sua utilização em áreas como química, física, biologia e computação possuem um potencial científico de investigação, visto que a construção de laboratórios virtuais neste tipo de ambiente enseja simular experiências e visualizações que muitas vezes são caras, complexas e/ou com risco de serem realizadas no mundo real.

Para a construção e implementação deste tipo de simulações, o uso de scripts de programação, por exemplo, na linguagem OSSL, uma das nativas do ambiente, pode ser utilizado em consonância com os objetos 3D disponíveis no mundo virtual. Agregado a isto, recursos multimídias também podem ser utilizados neste ambiente como forma de apoio ao processo de ensino e aprendizagem, no qual podem ser utilizados vídeos, slides, textos, questões e navegação em sites da internet diretamente no OpenSim.

Em virtude desta variedade de recursos educacionais que possam ser utilizados durante uma atividade de intervenção didática, o professor necessita obter formas de realizar o monitoramento e avaliação de cada usuário dentro do ambiente. Visto, que por meio da observação ou gravação de telas, este processo se torna oneroso e complexo, novas alternativas necessitam ser desenvolvidas para efetuar a coleta de dados que possam ser úteis ao processo de avaliação do docente.

Desta forma, o professor poderá ter a possibilidade de realizar uma análise mais detalhada dos dados coletados e processar informações que possam ajudá-lo a avaliar o aluno de forma mais coerente e precisa. A próxima seção deste artigo apresenta uma proposta que enseja contribuir para o melhor funcionamento deste processo de monitoramento e avaliação de usuários pelo docente em mundos virtuais.

\section{PROPOSTA DE MONITORAMENTO DOS ALUNOS NO OPENSIM}

O desenvolvimento desta proposta está centrada na necessidade do docente obter um monitoramento mais preciso e detalhado acerca das atividades educacionais realizadas pelos alunos dentro dos mundos virtuais, visto que tais tarefas podem ser executadas em qualquer momento e local de forma online. Os dados possíveis de serem coletados podem vir a auxiliar o professor em sua avaliação do desempenho de cada aluno e verificar como está sendo a experiência interativa experimentada por eles, por meio da análise quantitativa e qualitativa das informações disponíveis.

Com relação ao ponto de vista tecnológico desta proposta, foi selecionado o mundo virtual OpenSim por ser gratuito, open source, com vasta documentação no meio acadêmico e de conhecimento dos autores deste trabalho; para sua visualização, o viewer Singularity foi selecionado por atender às necessidades exigidas no desenvolvimento deste projeto; também foi utilizado um servidor Web para hospedar a aplicação Wamp Server, que inclui o software Apache, banco de dados MySQL para armazenar as informações provenientes do mundo virtual e a linguagem de programação PHP para realizar a criação dos scripts.

Levando em consideração este contexto, foi articulada uma proposta inicial que contemple alguns dos principais elementos que podem vir a auxiliar o professor em sua 
análise sobre os estudantes. É importante ressaltar que tais elementos foram escolhidos com base nos trabalhos analisados, conforme descrito na seção 2, assim como no conhecimento empírico dos autores deste trabalho, que tem como base diversas pesquisas realizadas anteriormente envolvendo mundos virtuais e sua aplicação no âmbito educacional.

Portanto, foram selecionados os seguintes pontos de verificação e coleta de dados dentro do mundo virtual:

- Sensores de presença para controle da assiduidade dos alunos;

- Sensores de interação dos alunos no ambiente;

- Sensores para as avaliações realizadas dentro do ambiente;

- Gravação do chat.

Listado os pontos de verificação desenvolvidos, se torna necessário esclarecer os detalhes referentes ao processo de criação e implementação dentro do mundo virtual. Tais sensores podem ser criados dentro do OpenSim utilizando a linguagem OSSL para realizar a programação desejada, na qual foram adicionadas esferas dentro ambiente e acopladas a elas os scripts criados, conforme pode ser visto na Figura 1.

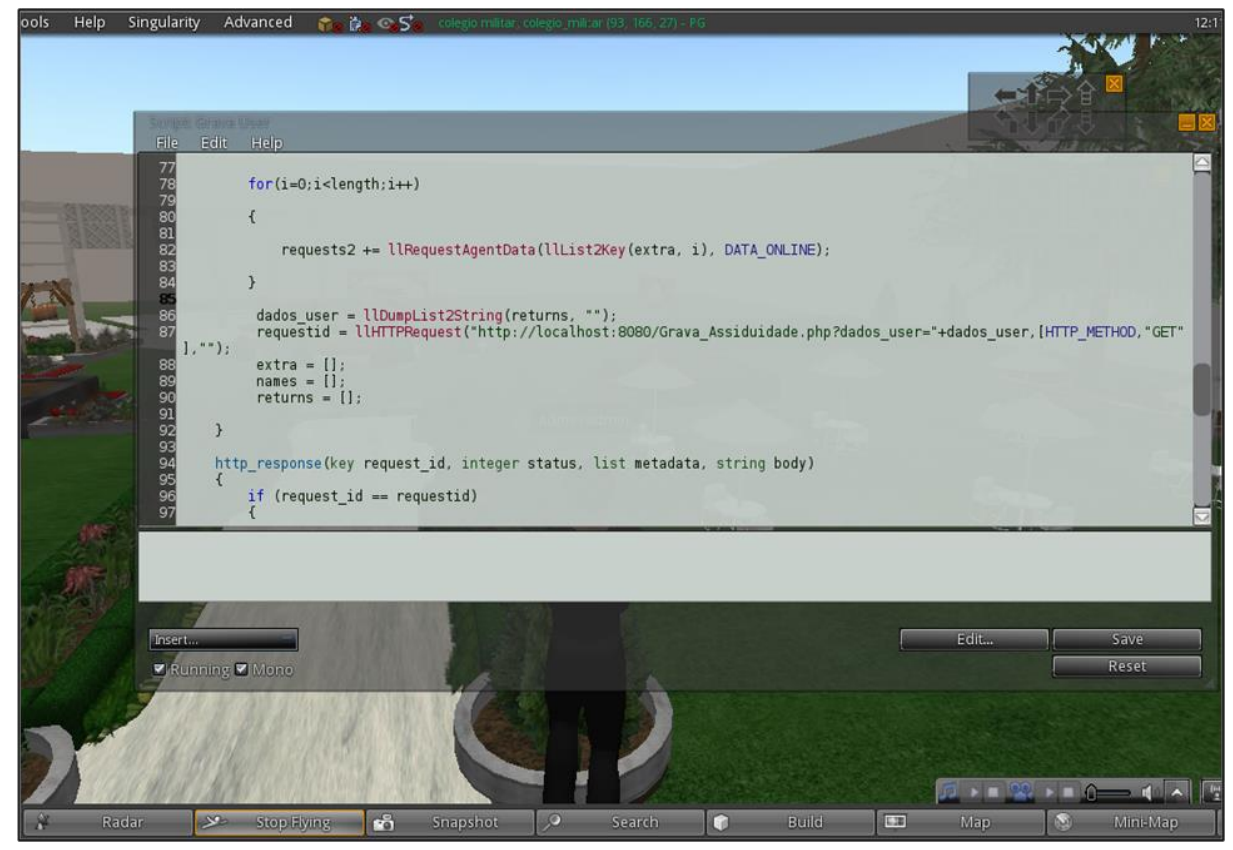

Figura 1 - Exemplo de script acoplado ao sensor de assiduidade no ambiente

Os scripts estão interligados à arquivos externos desenvolvidos utilizando a linguagem de programação PHP e estão localizados no servidor Web, em que a comunicação entre estes é realizada por meio dos comandos HTTP Request e Response. Portanto, o modo de funcionamento ocorre da seguinte maneira: os scripts no OpenSim captam os dados especificados anteriormente e enviam para os arquivos PHP que realizam o tratamento e armazenamento destes dados no banco de dados do OpenSim.

Tais dados podem ser atualizados conforme as informações vão sendo coletadas dentro do mundo virtual, fornecendo assim um monitoramento em tempo real e atualizado das interações realizadas pelos alunos no ambiente. Um laboratório educacional foi criado e instalado os diferentes sensores explicitados anteriormente. Neste espaço foram criadas cinco salas, que podem conter diversos tipos de mídias, e.g. vídeos, textos, slides, questões e simulações de objetos 3D, cuja área e conteúdos abordados neste espaço tratavam de ciência básica. 
Desta forma, o primeiro item a ser descrito são os sensores de presença para controle da assiduidade dos alunos, que por meio de um único sensor na região em que está localizado o laboratório educacional, efetua os registros de acesso e desconexão de cada usuário. Os dados armazenados em uma tabela denominada "presence_avatar" são o nome do aluno, a região que estava, o registro temporal de quando entrou no ambiente e também de quando se desconectou.

Além disso, este sensor indica se o usuário está online em tempo real. Portanto, o professor tem uma informação dinâmica dos usuários que estão interagindo com o mundo virtual, assim como pode realizar o controle de assiduidade dos alunos e fornecer presença ou falta na atividade com base nos registros de acesso ao ambiente armazenados nesta tabela do banco de dados.

Com relação aos sensores de interação dos alunos no ambiente, uma tabela denominada "record_activities" foi criada para armazenar dados referentes às interações dos alunos em cada sala do laboratório. Os dados armazenados são o nome do usuário e seu identificador único, o laboratório que foi visitado e cada sala que visitou. Também são armazenadas a data e hora de cada registro e os tipos de interações que o usuário realizou em cada sala.

Visto que diversos locais ou laboratórios podem ser criados no ambiente, a identificação de cada um permite fornecer uma visão mais clara para o professor, assim como dentro de cada um destes locais, a divisão em diferentes salas, por exemplo, sala de vídeos, slides, simulações, entre outros, permite um maior detalhamento por parte do professor em sua análise. O sensor analisa cinco tipos diferentes de interações do aluno (escrevendo, ausente, voando, andando ou parado), sendo armazenado o tempo total por dia que cada avatar ficou em cada uma destas posições.

É importante ressaltar que o tempo é coletado a cada intervalo de 15 segundos, sendo somado ao tempo já existente para aquele dia e no respectivo status. O uso deste indicador de status pode inferir se o usuário estava interagindo no ambiente ou não, por exemplo, se seu status está ausente, isto significa que ele está sem interagir no mundo virtual e o tempo que ficou nesta posição durante o dia é armazenado no banco de dados.

Descritos os sensores relacionados ao registro das interações dos usuários no mundo virtual, outro ponto considerado essencial está centrado na realização de avaliações dentro do mundo virtual. Para contemplar esta necessidade, foi desenvolvido um sistema de avaliação dentro do mundo virtual, que pode ser visualizado na Figura 2, no qual o usuário responde às perguntas e estas são armazenadas diretamente em uma tabela denominada "record_grades" no banco de dados. O professor pode visualizar o nome do aluno que realizou a avaliação, as alternativas que selecionou e quais questões acertou ou errou, o seu desempenho total, o laboratório onde respondeu esta avaliação e o registro temporal. 


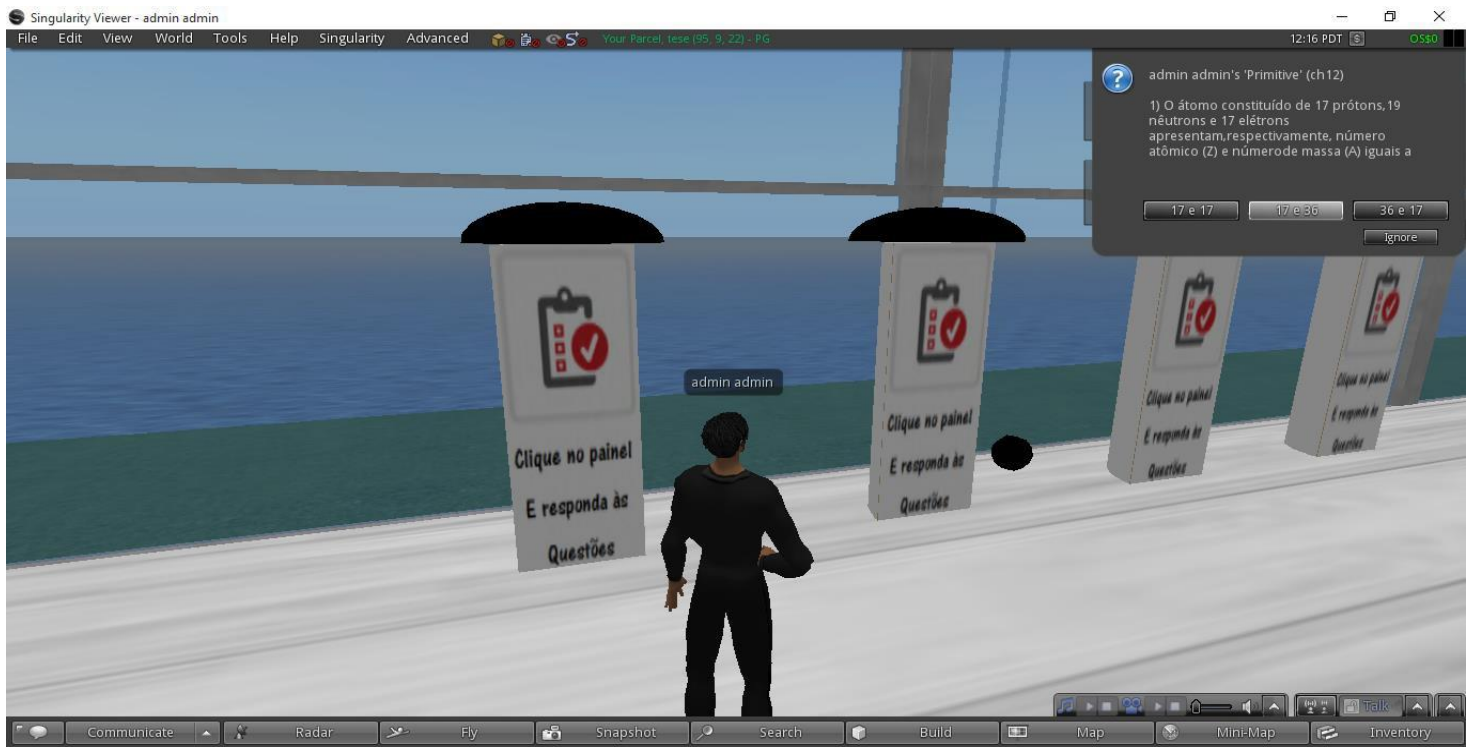

Figura 2 - Exemplo de questões sendo respondidas no mundo virtual

Os tipos de questões suportadas são: múltipla escolha com feedback imediato ao aluno sobre seu desempenho em cada questão e total; comparação de respostas dissertativas por meio de termos chaves que devam estar presentes, sendo fornecido o feedback imediato ao aluno se sua resposta contém os termos chaves correspondentes; e, respostas dadas pelo usuário de forma dissertativa que estarão à disposição do professor para avaliar manualmente. Tal mecanismo provê ao professor um suporte adequado para a realização de atividades básicas de avaliação com os alunos diretamente no mundo virtual, obtendo de forma automática o registro e posterior visualização dos resultados de cada aluno. Com relação ao aluno, a realização destas tarefas diretamente no mundo virtual está focalizada na manutenção do aspecto de imersão e interatividade que são propostos nesse tipo de ambiente.

Por fim, o último recurso implementado nesta proposta está relacionado ao chat do mundo virtual. Foi utilizado um script presente no site "Outworldz.com", cujo código é aberto e disponível para ser utilizado e modificado por qualquer pessoa, que realiza o armazenamento de todas as conversas efetuadas entre os usuários do mundo virtual. Pequenos ajustes foram feitos, sendo o registro do chat armazenado em um arquivo de texto e permitida sua visualização em uma página HTML, na qual está contido o nome do usuário e a data e hora que escreveu o texto.

Tal recurso é importante para os docentes que queiram analisar a interação textual entre os alunos e analisar o nível de colaboração em um determinado período de tempo. Técnicas relacionadas à mineração de textos também poderiam ser aplicadas para investigar padrões e peculiaridades nos registros coletados no mundo virtual. Efetuada tais descrições, são apresentados alguns resultados parciais obtidos com usuários reais.

\subsection{Resultados Preliminares}

Para efetuar os testes iniciais desta proposta, foi utilizado três turmas compostas cada uma por 25 alunos da sexta série e realizado um encontro de 50 minutos com cada turma. $\mathrm{O}$ foco do encontro esteve centralizado na apresentação do mundo virtual e teve como objetivo viabilizar o acesso dos alunos pela primeira vez no ambiente.

As atividades desempenhadas no ambiente foram de personalização do avatar de cada um e a visitação do laboratório descrito anteriormente com todos os sensores ativados. Eles deveriam interagir de forma inicial com os recursos multimídias presentes 
nas salas e entender como se dava o funcionamento de cada, ressaltando que estas atividades não tiveram um enfoque educacional, mas sim de validação do ponto de vista tecnológico.

Como resultados obtidos, foi possível confirmar que todos os sensores funcionaram corretamente, levando em consideração que os dados referentes ao controle de acesso dos usuários operaram de forma correta. Com relação à coleta de dados das interações dos usuários, a Figura 3 apresenta uma parte dos dados armazenados, sendo contabilizado um total de 348 registros. Como pode ser visualizado na Figura 3, na qual foram omitidos os nomes dos avatares de forma a manter o anonimato, os registros ocorrerem de forma correta e não foi detectado nenhum erro de gravação nos dados coletados. Além do identificador do avatar do aluno e seu nome, foi armazenado o nome do laboratório onde ocorreu a interação (Laboratório 02), assim como o último registro temporal feito na tabela.

Importante ressaltar que existiam cinco tipos de comportamentos que poderiam ser identificados, onde na figura pode se visualizar as interações "voando" e "andando", com o respectivo tempo que o usuário ficou realizando esta ação. No caso da primeira linha, pode se perceber que o aluno permaneceu o total de um minuto e quinze segundos naquele dia dentro da sala de vídeos realizando a ação de voar.

Assim como, na segunda linha podemos ver que o usuário permaneceu três minutos e sessenta segundos voando dentro da sala de questões, lembrando que ele poderia estar interagindo com objetos nesta posição e também se movimentando. Um aspecto importante a ser esclarecido é que com estas informações, o professor pode inferir se o usuário estava interagindo no ambiente.

Esta análise pode ser feita levando em conta que um dos possíveis status que o aluno pode permanecer é "ausente", ou seja, este estado indica com precisão que ele ficou com o visualizador aberto, porém não estava interagindo no ambiente. Por exemplo, caso o aluno minimize o visualizador e fique interagindo em seu computador, o seu avatar entrará automaticamente no status "ausente" até que ele volte a interagir com seu personagem no ambiente.

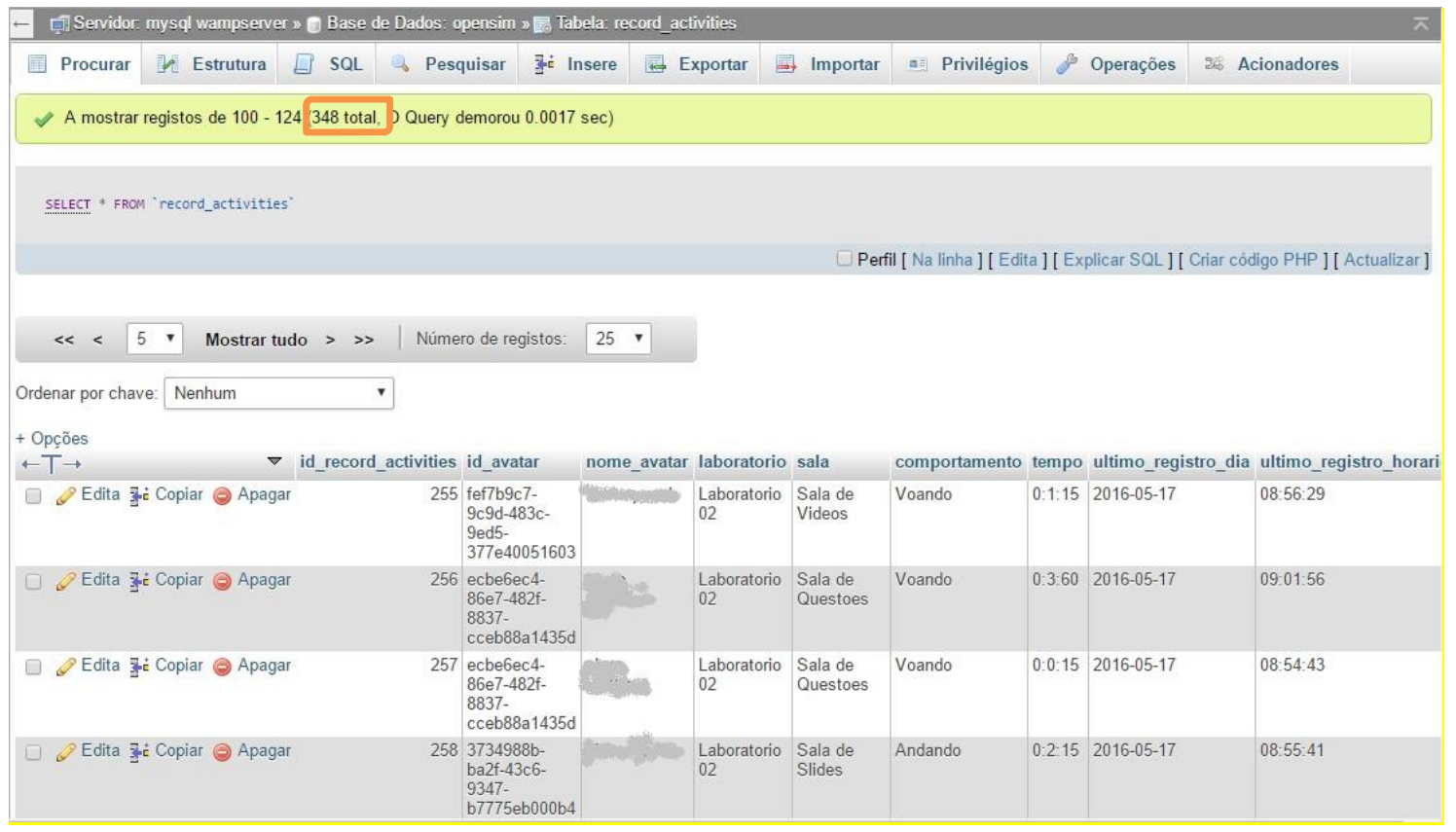

Figura 3 - Dados obtidos a partir das interações dos usuários nos testes realizados 
Os demais recursos presentes no laboratório também operaram de forma correta, como o registro das conversas efetuadas por eles no chat e o uso do objeto de avaliação para responder às questões de múltipla escolha que haviam sido disponibilizadas sobre o conteúdo de ciência básica. Importante ressaltar que não houve o registro de nenhuma crítica dos alunos quanto a erros que poderiam ter ocorrido durante as interações no mundo virtual.

\section{CONCLUSÃO}

Com a utilização das tecnologias no âmbito educacional, novos paradigmas e metodologias de ensino foram introduzidos. A utilização dos mundos virtuais ganhou força no decorrer dos últimos anos no campo educacional, sendo desenvolvidas diversas iniciativas com o objetivo de possibilitar a integração destes recursos com as atividades educacionais realizadas pelos alunos em diferentes áreas de atuação.

O monitoramento e avaliação no mundo virtual dos alunos se caracteriza como um problema importante, visto que o docente não consegue monitorar o tempo inteiro e em todos lugares as interações realizadas pelos usuários. Levando em consideração esta problemática, foi proposto neste artigo o desenvolvimento de um conjunto de recursos no OpenSim para realizar a coleta de dados que possam ajudar o professor a verificar quais foram as atividades realizadas neste ambiente.

Os resultados parciais apresentados neste artigo mostram os primeiros passos desenvolvidos para realizar o monitoramento em tempo real dos alunos dentro mundo virtual. Por meio dos testes efetuados com três grupos diferentes de usuários, foi possível constatar que houve a efetiva coleta dos dados referentes ao acesso dos usuários no mundo virtual, suas ações e interações em cada sala, registros das conversas no chat, assim como o processo de avaliação ocorreu normalmente.

Tais informações podem ser consideradas de grande valia para o professor, visto que ele tem a sua disposição um conjunto de dados que podem ser manipulados e analisados para obter um maior controle do desempenho de cada aluno durante o decorrer do semestre, assim como identificar possíveis estudantes que não tenham realizado as atividades e/ou obtido um desempenho ruim na disciplina, para que possam ser aplicadas ações corretivas em tempo hábil. Desta forma, pode se considerar satisfatória a primeira etapa de testes realizados no ambiente, ressaltando que somente a viabilidade e correto funcionamento dos recursos foram testados neste processo.

As próximas etapas contemplam o aprimoramento destes recursos desenvolvidos e a criação de novas funcionalidades que possam auxiliar o professor, além de realizar testes robustos com o ambiente no sentido de avaliar o ponto de vista educacional, ou seja, se estes recursos auxiliaram o professor a melhorar o processo de ensino e aprendizagem durante todo o semestre. Também se tem como objetivo fornecer uma maior autonomia para o professor, no sentido de disponibilizar o suporte tecnológico necessário para que eles possam realizar, pelo menos em parte, a criação de atividades educacionais dentro do mundo virtual por conta própria.

\section{REFERÊNCIAS BIBLIOGRÁFICAS}

AMARAL, E; ÁVILA, B. G; TAROUCO, L. M. R. Aspectos teóricos e práticos da implantação de um laboratório virtual no OpenSim. Anais do $\mathbf{2 3}^{\circ}$ Simpósio Brasileiro de Informática na Educação, 2012. 
ANTUNES, R., MORGADO, L., MARTINS, P., \& FONSECA, B. Gestão do acompanhamento de actividades de alunos em mundos virtuais: estudo exploratório no Second Life. Conferência IADIS Ibero-Americana WWW/Internet, p. 1-5, 2012. BOUDA, T. User-centered Design and Evaluation of Virtual Worlds. Experiential Learning in Virtual Worlds: 2nd Global Conference, 2012.

BULU, S. T. Place presence, social presence, co-presence, and satisfaction in virtual worlds. Computers \& Education, n. 58, p. 154-161, 2012.

CAMILLERI, V., FREITAS, S. DE, MONTEBELLO, M., \& MCDONAGH-SMITH, P. A case study inside virtual worlds: use of analytics for immersive spaces. Proceedings of the Third International Conference on Learning Analytics and Knowledge, p. 230-234, 2013.

CARMO, F. M. do. Mundo Virtual 3D em plataforma aberta como interface para ambientes de aprendizagem. Tese de Doutorado, Escola Politécnica da Universidade de São Paulo, 2013.

FALCADE, A., KRASSMANN, A., HERPICH, F., FALCADE, L., FONTOURA, L. M., MEDINA, R. D. Metodologia de Avaliação de Mundos Virtuais Educacionais: uma proposta segundo critérios tecnológicos e pedagógicos. RENOTE, v. 12, n. 1, 2014.

MADEIRA, A. J. da S. Controle de assiduidade em aulas efetuadas no mundo virtual Second Life. Dissertação de Mestrado em Informática, 2008.

NUNES, F. B., VOSS, G. B., HERPICH, F., MÜHLBEIER, A. R. K., POSSOBOM, C. C., MEDINA, R. D. Viewers para ambientes virtuais imersivos: uma análise comparativa teórico-prática. RENOTE, v. 11, n.1, 2013.

RAFALSKI, J. P., JUNIOR, R. R. M. V., SILVA, C. A. S. Mundos Virtuais como Suporte à Aprendizagem - Uma Avaliação na Implementação de Projetos de Aprendizagem. Renote, v. $12 \mathrm{n}^{\mathrm{o}} 1,2014$.

REIS, S. C., L. A. J. M., MATTIELLO, L. English 3D: Uma proposta de curso de inglês a distância no projeto LabEOn. Nuevas Ideas en Informática Educativa (TISE), p. 1-7, 2013.

TIBOLA, L. R., TAROUCO, L. M. R. Rastreamento de interações em laboratórios educacionais nos mundos virtuais 3d para identificação de engajamento. RENOTE, v. 13, $\mathrm{n}^{\mathrm{o}} 2,2015$.

YILMAZ, R. M., BAYDAS, O., KARAKUS, T., \& GOKTAS, Y. An examination of interactions in a three-dimensional virtual world. Computers \& Education, 88, 256$267,2015$. 